\title{
Adsorption of PET on concrete nano-structures: an in-silico assay
}

\author{
L. Bárcenas ${ }^{1}$, F. Aguilera-Granja ${ }^{2}$, G. Ramírez-García ${ }^{3}$, E. Díaz-Cervantes ${ }^{1^{*}}$ \\ ${ }^{1}$ Departamento de Alimentos, Centro Interdisciplinario del Noreste (CINUG), Universidad de Guanajuato, 37975 Tierra \\ Blanca, Guanajuato, México. tl.barcenasramirez@ugto.mx* e.diaz@ugto.mx* \\ ${ }^{2}$ Instituto de Física, Universidad Autónoma de San Luis Potosí, 78000 San Luis Potosí, SLP, México \\ ${ }^{3}$ Cátedras CONACYT-Centro de Investigación en Química Aplicada, COITTEC, 140, Blvd. Enrique Reyna, Saltillo, \\ Coahuila 25294, México.
}

\begin{abstract}
The present work analyses the first approach of the use of PET as a component of a sustainable alternative to generate an enforced concrete. Through the computational study of one nanostructured concrete model and two monomers PET system, the adsorption energies of PET on concrete were evaluated in the present work, which was exergonic $(-7.67 \mathrm{eV})$ and therefore can be formulated as a perspective a sustainable concrete using one of the principal waste in the world, applied to the construction industry.
\end{abstract}

Keywords: PET, Concrete, Nano-structures, Adsorption-energy, DFT

\section{Introduction}

Nowadays the earth suffers the highest levels of pollution of all times, for this reason, the need to reuse and avoid the contaminant resources is key to solve this urgent problem. In this order, highlights that polyethylene terephthalate (PET) is a thermoplastic resin, at an industrial level it is obtained by a polymerization reaction, which has been tried to degraded in several ways[1,2], including biological treatments, however, this remains to be a problem and is latent the need to reuse it.

On the other hand, in the construction industry, one of the main used materials is the concrete, 11 billion tons[3] per year, which can be doped with some waste materials to decrease the pollution and at the same time costs in this industry. The state of the art about the chemical structure and composition of concrete specify that the product results from the hydration of common Portland cement, which is a porous matrix consisting of several crystallites embedded in a poorly crystallized phase, the so-called calcium-silicate-hydrate $(\mathrm{C}-\mathrm{S}-\mathrm{H})$ gel. This $\mathrm{C}-\mathrm{S}-\mathrm{H}$ gel is the most important hydration product. It constitutes about $50-70 \%$ of the fully hydrated cement paste and is responsible for most of the engineering properties of cement-based materials[4].

Moreover, the CSH concrete gel has a variable stoichiometry, generally characterized by its water content and inclusive calcium-silicon constituents. At the same time the structure, elastic, and mechanic properties have been several reported by Ayuela and co-workers since a few years ago[5-8]. Other recent studies proposed the chemical structure of some cement clusters studied at the DFT level[9].

Based on the above-mentioned studies, the present work aims is to study the possible structural conformation of a sustainable concrete model, based on a nanoparticle structure of concrete linked to one of the most pollution resources, PET.

\section{Results and discussion}

The results obtained about the concrete nano-cluster structure is depicted in Fig. 1, which shows the optimized structure of our proposed concrete nano-model.

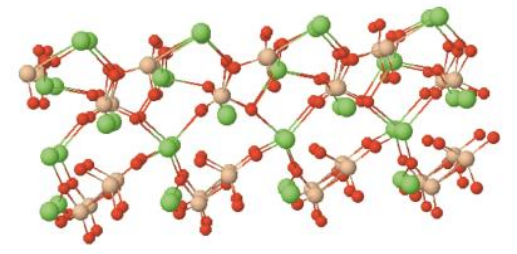

Fig. 1: Optimized structure of nanostructured concrete. 
Fig. 1 shows the stoichiometric proportions selected to perform a concrete structure, which is based on the proposed model by Ayuela and co-workers. Note that the condensed formula is $\mathrm{O} 15 \mathrm{Si} 4 \mathrm{Ca} 5$, which is the remainder after mix all the over components of concrete.

Once the concrete nanostructure was established and the PET model was optimized, the next step was to model the adsorption of PET on the concrete surface, which has been made using the DFT formalism, as will specify in the methods section. Fig. 2 shows the optimized composite (Concrete-PET system), which depicts that the PET remains in longdistance respect to the concrete cluster, but highlights that the adsorption energy is favorable, being this of $-7.67 \mathrm{eV}$.

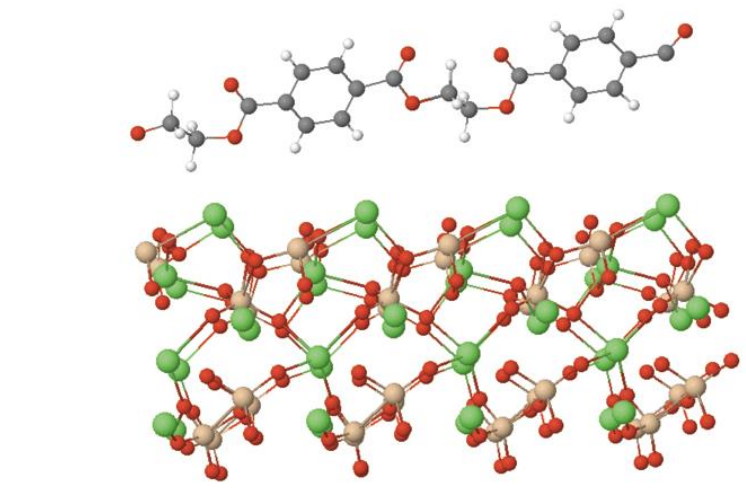

Fig. 1: Optimized

$$
\begin{gathered}
\text { Concrete }+ \text { PET } \rightarrow \text { Composite }_{P E T-\text { Concrete }}(1) \\
E_{\text {ads }}=E_{\text {comp }}-\left(E_{P E T}+E_{\text {conc }}\right)
\end{gathered}
$$

The adsorption energy was computed using the Eq (1) and Eq (2), and as has been mentioned above, this was exergonic and therefore allows the PET adsorption on concrete, which can be used as evidence and a first approach to the possible formation of the PET-concrete composite.

\section{Computational methods}

The concrete nanostructure was modeled based on the model proposed by Ayuela and co-workers[4]. The concrete and the PET molecules were modeled using the Avogadro package[10] and optimized through the PBE[11] functional using the SIESTA software.[12]

Finally, the composite was modeled and optimized at the same level as their components and based on the methodology performed by some of us[13]. The molecules were visualized using the Jmol software.[14]

\section{Conclusions}

Through the computational study of one nanostructured concrete model and two monomers PET system, the adsorption energies of PET on concrete were evaluated in the present work, which was exergonic and therefore can be formulated as a perspective a sustainable concrete using one of the principal waste in the world, applied to the construction industry.

\section{Acknowledgements}

We are grateful to the Laboratorio Nacional de Caracterización de Propiedades Fisicoquímicas y Estructura Molecular (UG-UAA-CONACYT, Project: 123732) for the computing time provided at the PIPILA cluster.

\section{References}

[1] T. Sang, C. J. Wallis, G. Hill, and G. J. P. Britovsek, "Polyethylene terephthalate degradation under natural and accelerated weathering conditions," Eur. Polym., vol. 136, p. 109873, 2020.

[2] D. Moog, J. Schmitt, J. Senger, J. Zarzycki, K.-H. Rexer, U. Linne, et al., "Using a marine microalga as a chassis for polyethylene terephthalate (PET) degradation," Microb. Cell Fact., vol. 18, p. 171, 2019. 
[3] H. Singh and K. Talwar, "Evaluation of Sulphate Resistance \& Effects of Glass Powder as A Supllementary Cementitious Material on the Performance of High Strength Mortar," Int. J. Eng. Res. Tech, vol. 9, pp. 346-353, 2020.

[4] P. Rejmak, J. S. Dolado, M. J. Stott, and A. Ayuela, "29Si NMR in Cement: A Theoretical Study on Calcium Silicate Hydrates," J. Phys. Chem. C, vol. 116, pp. 9755-9761, 116.

[5] H. Manzano, R. González-Teresa, J. S. Dolado, and A. Ayuela, "X-ray spectra and theoretical elastic properties of crystalline calcium silicate hydrates: comparison with cement hydrated gels," Mater. Construc., vol. 60, pp. 7-19, 2010.

[6] H. Manzano, J. S. Dolado, and A. Ayuela, "Elastic properties of the main species present in Portland cement pastes," Acta Mater., vol. 57, pp. 1666-1674, 2009.

[7] H. Manzano, J. S. Dolado, A. Guerrero, and A. Ayuela, "Mechanical properties of crystalline calciumsilicate-hydrates: comparison with cementitious C-S-H gels," Phys. Status Solidi A, vol. 204, pp. 1775$1780,2007$.

[8] H. Manzano, A. Ayuela, and J. S. Dolado, "On the formation of cementitious C-S-H nanoparticles," $J$ Computer-Aided Mater. Des., vol. 14, pp. 45-51, 2007.

[9] C. Qi, L. Liu, J. He, Q. Chen, L.-J. Yu, and P. Liu, "Understanding Cement Hydration of Cemented Paste Backfill: DFT Study of Water Adsorption on Tricalcium Silicate (111) Surface," Minerals, vol. 9, p. 202, 2019.

[10] M. D. Hanwell, D. E. Curtis, D. C. Lonie, T. Vandermeersch, E. Zurek, and G. R. Hutchison, "Avogadro: an advanced semantic chemical editor, visualization, and analysis platform," J. Cheminf., vol. 4, p. 17, 2012.

[11]J. P. Perdew, K. Burke, and M. Ernzerhof, "Generalized Gradient Approximation Made Simple," Phys. Rev. Lett., vol. 77, pp. 3865-3868, 1996.

[12] J. M. Soler, E. Artacho, J. D. Gale, A. García, J. Junquera, P. Ordejón, et al., "FREE ARTICLE

The SIESTA method for ab initio order-N materials simulation," J. Phys. Cond. Matt., vol. 14, p. 2745, 2002.

[13] G. Ramírez-García, E. D. a. Cervantes, O. Mounzer, E. D. 1. Rosa, T. Loṕez-Luke, and F. N. d. 1. Cruz, "A Turn-On Luminescence Method for Phosphate Determination Based on Fast Green-Functionalized ZrO2:Yb,Er@ZrO2 Core@Shell Upconversion Nanoparticles," Anal. Chem., vol. 91, pp. 14657-14665, 2019.

[14]"Jmol: an open-source Java viewer for chemical structures in 3D," ed. 